\title{
Evaluation of Protocol Uniformity Concerning Laparoscopic Cholecystectomy in The Netherlands
}

\author{
Linda S. G. L. Wauben · Richard H. M. Goossens • \\ Daan J. van Eijk · Johan F. Lange
}

Published online: 28 January 2008

(C) The Author(s) 2008

\begin{abstract}
Background Iatrogenic bile duct injury remains a current complication of laparoscopic cholecystectomy. One uniform and standardized protocol, based on the "critical view of safety" concept of Strasberg, should reduce the incidence of this complication. Furthermore, owing to the rapid development of minimally invasive surgery, technicians are becoming more frequently involved. To improve communication between the operating team and technicians, standardized actions should also be defined. The aim of this study was to compare existing protocols for laparoscopic cholecystectomy from various Dutch hospitals.

Methods Fifteen Dutch hospitals were contacted for evaluation of their protocols for laparoscopic cholecystectomy. All evaluated protocols were divided into six steps and were compared accordingly.

Results In total, 13 hospitals responded -5 academic hospitals, 5 teaching hospitals, 3 community hospitals—of which 10 protocols were usable for comparison. Concerning the trocar positions, only minor differences were found. The concept of "critical view of safety" was represented in just one protocol. Furthermore, the order of clipping and cutting the cystic artery and duct differed. Descriptions of instruments and apparatus were also inconsistent.

Conclusions Present protocols differ too much to define a universal procedure among surgeons in The Netherlands.
\end{abstract}

L. S. G. L. Wauben $(\bowtie) \cdot$ R. H. M. Goossens · D. J. van Eijk Faculty of Industrial Design Engineering, Delft University of Technology, Landbergstraat 15, 2628, CE, Delft, The Netherlands

e-mail: 1.s.g.l.wauben@tudelft.nl

L. S. G. L. Wauben · J. F. Lange

Department of Surgery, Erasmus MC, Dr. Molewaterplein 40,

3015, GD, Rotterdam, The Netherlands
The authors propose one (inter)national standardized protocol, including standardized actions. This uniform standardized protocol has to be officially released and recommended by national scientific associations (e.g., the Dutch Society of Surgery) or international societies (e.g., European Association for Endoscopic Surgery and Society of American Gastrointestinal and Endoscopic Surgeons). The aim is to improve patient safety and professional communication, which are necessary for new developments.

\section{Introduction}

Laparoscopic cholecystectomy is the most performed and acknowledged minimally invasive operation in The Netherlands $(15,000$ laparoscopic cholecystectomy procedures in 2005) [1-4]. Still, a regular complication of this procedure remains iatrogenic biliary tract injury [1,5-7]. The most common cause of serious injuries is misidentification of the anatomy in general and misidentification of the cystic duct in particular [1, 6-11]. Consensus exists that complete dissection of Calot's triangle reduces the incidence of bile duct injury [7, 8, 10-12]. This is especially achieved by the technique of "critical view of safety" of Strasberg in which Calot's triangle is completely unfolded by mobilizing the gallbladder neck from the gallbladder bed of the liver before transecting the cystic artery and duct (Fig. 1).

Consequently, the availability of a uniform, standardized protocol such as those used in other technical high-risk fields (e.g., aviation, nuclear industry, oil industry) would be advantageous for patient safety [13]. A protocol is a formal set of guidelines usually consisting of actions to be 


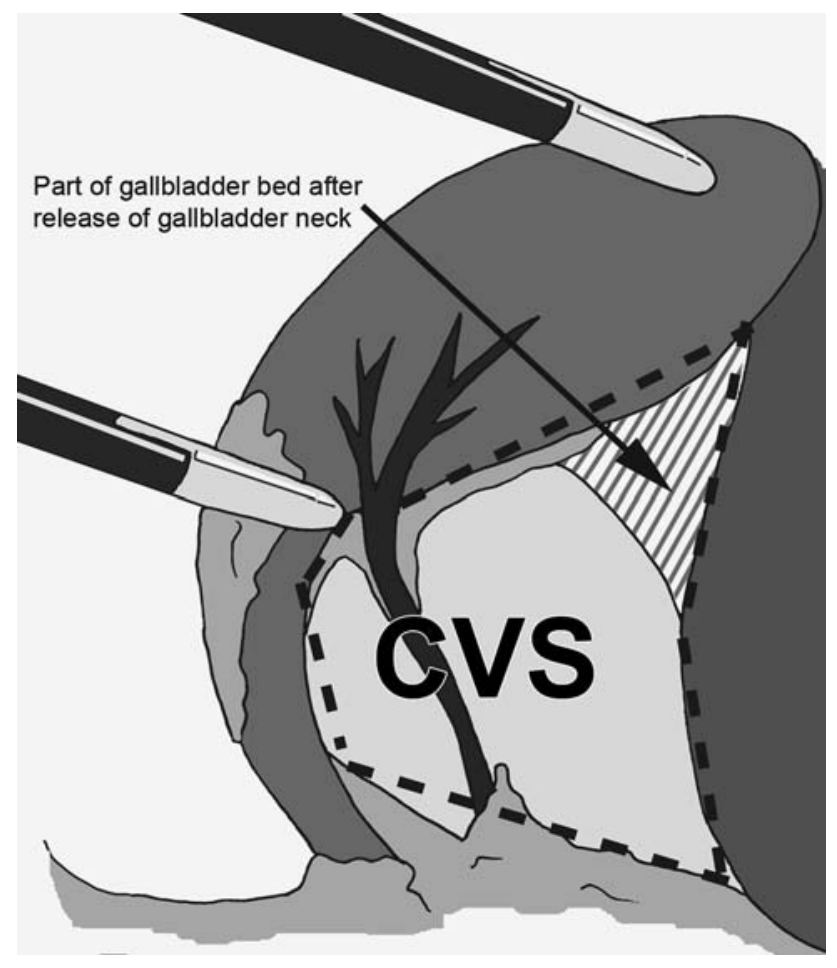

Fig. 1 Critical view of safety (CVS) for laparoscopic cholecystectomy

performed, leading to a specific end result. An example are the protocols used in aviation (checklists) in which crosschecking the crucial steps is deployed to guarantee safety [14].

Successful surgery and enhancing the safety of health care also depends on effective teamwork. Because of the growing complexity and continuing developments in surgical operations, the entire operating team (surgeons, nurses, anesthesiologists, assistants, residents) should be more actively involved during surgery $[15,16]$. A uniform standardized protocol could contribute to the shared understanding of their roles, tasks, and objectives throughout the surgical process as well as enhancing surgical education and training.

Furthermore, owing to the fast growth of minimally invasive surgical techniques accompanied by the increased use of more complex apparatus and instruments, technicians are no longer a supplier of equipment but represent an important source of information [17-19]. Because technical principles from industry play a substantial role in improving medical treatment, a major point of interest is the implementation and integration of technical quality systems in health care.

The Dutch Society of Surgery demands that each surgical department of a Dutch training hospital has a protocol for operative procedures. The use of the protocol is obligatory and is globally checked by means of site visits by the Dutch Society of Surgery every $1-5$ years. Although no specific requirements are provided by the Society, in this study a protocol is defined as the steps to perform a successful operation (operation method), whether to include the necessary apparatus and instruments.

The aim of this study was to evaluate the existing protocols for laparoscopic cholecystectomy from various hospitals in The Netherlands by comparing the described steps (actions).

\section{Materials and methods}

\section{Participants}

In January 2006, a total of 15 surgical departments of Dutch hospitals were selected from the (2006) Dutch database of Wauben et al., with a special focus on laparoscopic surgery [20]. In total, 6 (of 8) academic hospitals, 6 (of 53) teaching hospitals, and 3 (of 33) community hospitals were contacted. By means of a letter, the contacted surgeons were requested to send the most recent protocol for laparoscopic cholecystectomy; or in case no protocol was available, participants were requested to inform the authors of its absence.

\section{Technical information}

The protocols were divided into six steps based on the "Best Practice for Laparoscopic Cholecystectomy 2006," which was drawn up by order of the Dutch Society of Surgery. The steps are (A) introduction of trocars; (B) exploration of the abdomen; (C) opening of the peritoneal envelope; (D) mobilization of the infundibulum and "critical view of safety"; (E) clipping and cutting the cystic artery and duct; and $(\mathrm{F})$ performing retrograde cholecystectomy and terminating the procedure. The protocols for laparoscopic cholecystectomy of the various hospitals were compared in which the chronologic action order was maintained. Initially, instructions, warnings, remarks, and the use of specific instruments were not included in the comparison.

\section{Results}

A total of 13 hospitals responded to the request: 5 academic hospitals (A1-A5), 5 teaching hospitals (T1-T5), and 3 community hospitals (C1-C3) (Table 1). Various documents were received: best practice, protocols $(n=4)$, instructions $(n=3)$ and operative reports $(n=2)$. In this study, "instruction" meant a document in which the gallbladder and biliary tract were described including 
Table 1 Response and properties of the protocols for laparoscopic cholecystectomy

\begin{tabular}{lllllll}
\hline Protocol & Hospital & Type & Author mentioned & Edition/update & Reference & Layout \\
\hline A1 & Academic & Best practice & Yes & December 2005 & Yes & Text in paragraphs \\
A2 & & Protocol & Yes & November 12, 2003 & - & Step by step \\
A3 & & Instruction & - & - & - & Step by step \\
A4 & & Instruction & - & - & - & Step by step \\
A5 & & Instruction different procedure & N/A & N/A & N/A & N/A \\
T1 & Teaching & Protocol & - & - & - & One text \\
T2 & & Operative report & - & - & - & One text \\
T3 & & Instruction & - & April 2003 & Step by step \\
T4 & & Protocol & - & December 2005 & - & Step by step \\
T5 & & No protocol & N/A & N/A & N/A \\
C1 & Community & Operative report & Yes & June 3, 2003 & - One text \\
C2 & & Protocol & N/A & N/A & Step by step \\
C3 & & No protocol & & N/A & N \\
\hline
\end{tabular}

N/A: not available

deviations, surgical abnormalities, and surgical procedures, including laparoscopic cholecystectomy. The term "operative report" included a document used to compile the report after the procedure has been performed. The predescribed text section was then interpreted as the protocol. This article refers to all received documents as "protocol."

Three responses could not be used because hospitals T5 and $\mathrm{C} 3$ did not have a protocol and hospital A5 sent a protocol concerning a different procedure (Table 1). From the 10 remaining protocols, only A1 ("Best Practice") included references, and only three protocols stated the author. Furthermore, two protocols were of a recent date $(<$ 1 year old), and three were between 2.5 and 3.0 years old. Finally, the layout of the protocols differed: A1 described the actions in a single text subdivided into paragraphs; T12 and $\mathrm{C} 1$ described the actions in a single text; and the remaining protocols described the actions step by step. The results below are discussed according to the six steps.

Figure 2 shows the trocar positions (step A). The position of the optical trocar for $\mathrm{C} 2$ and A2 differed. They were placed above the umbilicus and in the umbilicus, respectively. T1 described the use of the Hasson trocar but did not describe a specific location. Except for A4 (no position indication), the positions of the epigastric trocars did not differ. Deviant position C2 was described by means of a graph. Three groups could be distinguished for the position of the working trocars. First, subcostally at the level of the axillary line (A3, T3, C1) and right at umbilical level (T4 and C2). Second, subcostally right (A2, T2, T4) and finally at the midclavicular line (A2-3, T2-3, C1). Protocols A1, A4 and T1 did not describe a specific position for the working trocars. C2 used, as opposed to all other protocols, only one working trocar, indicating a total of three instead of four trocars.

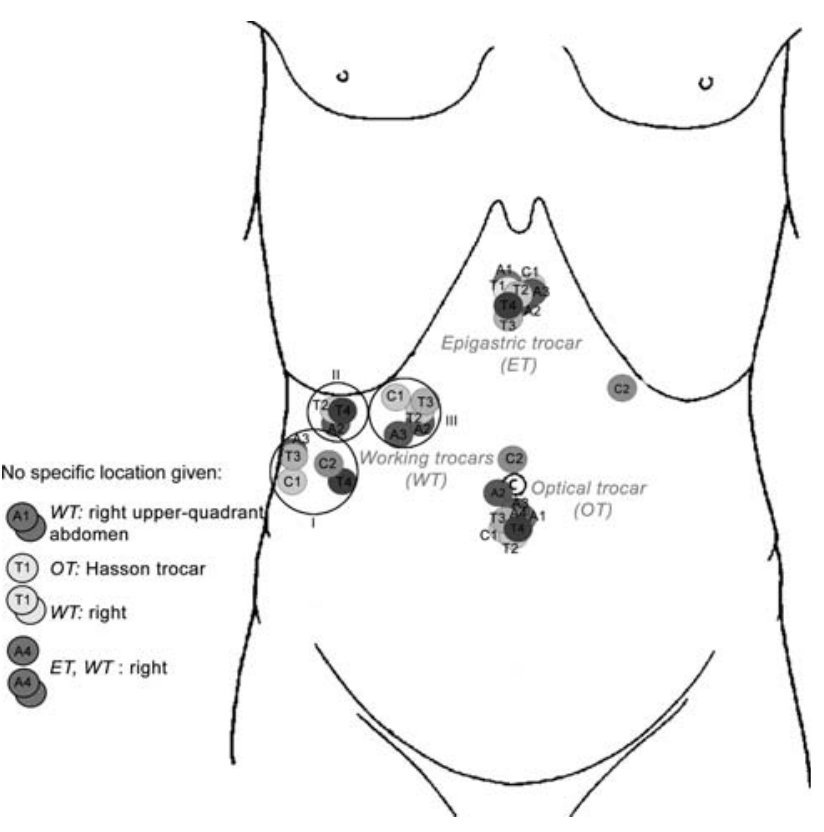

Fig. 2 Trocar positions

Table 2 gives a brief overview of the steps in the protocols. In step $B$ (exploration of the abdomen) only protocols A1, A2, and T1 explicitly described the inspection of the abdomen and gallbladder. Protocol A1 also described the identification of Rouvière's sulcus.

In step $C$ (opening the peritoneal envelope), grabbing the fundus (top) of the gallbladder is described by all protocols, except A3. However, protocols A4, T4, and C2 did not give an exact description of the actions ("adjust by positioning gallbladder," "retract gallbladder," and "grab gallbladder," respectively). Opening the peritoneum was described by protocols A1, A4, T1, and T3-4. 
Table 2 Steps from the protocols for laparoscopic cholecystectomy

\begin{tabular}{lllllllll}
\hline Steps & A1 A2 A3 A4 T1 T2 T3 T4 C1 C2 Identical steps \\
(no.)
\end{tabular}

(A) Introduction of trocars
1. Optical trocar
2. Inspect abdomen/GB
3. Incisions for trocars
4. Epigastric trocar
5. Working trocar(first)
6. Working trocar(second)
7. Epigastric trocar
8. Introduce instruments

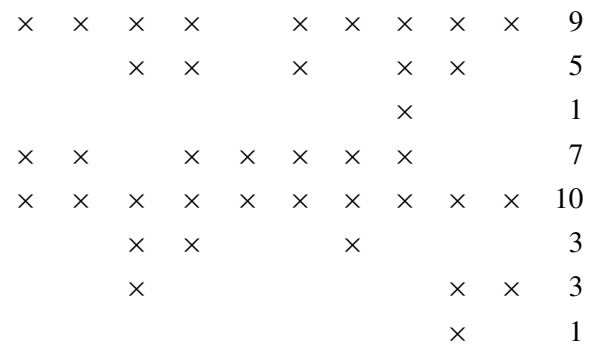

(B) Exploration of abdomen

9. Inspect abdomen/GB

10. Identify Rouvière's sulcus

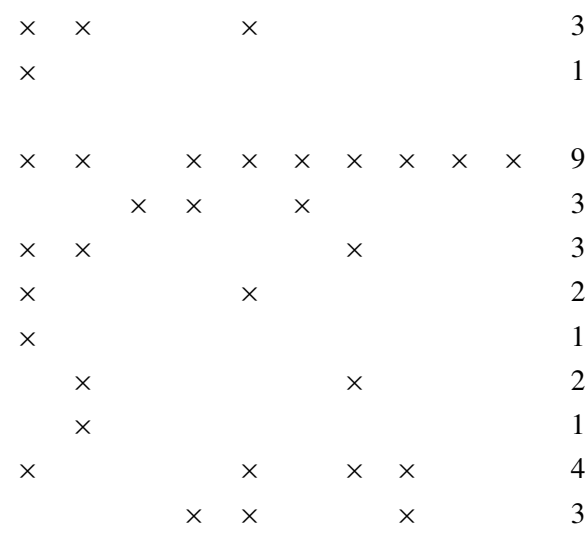

(C) Opening the peritoneal envelope

11. Grab fundus/top GB

12. Adjust/position GB

13. Apply traction cranially

14. Grab infundibulum

15. Tighten caudoventrally

16. Identify Hartmann's pouch

17. Apply traction laterally and somewhat caudally

18. Open peritoneum

19. Open peritoneum right side/in ligamentum hepatoduodenale, dissect tissue around arteria and ductus

(D) Mobilize the infundibulum and CVS

20. Dissect $\Delta$ Calot

21. Establish CVS by mobilizing infundibulum GB approx. one-third GB length $\times$ from GB bed of the liver

22. Dissect ductus

23. Dissect arteria

24. Dissect bottom edge GB and dissect $\Delta$ Calot

25. Exclude abberant right ductus hepaticus

(E) Clip and cut cystic artery and duct

26. Clip arteria

27. Cut arteria

28. Check CVS and Rouvier's sulcus

29. Dissect ductus

30. Dissect $\Delta$ Calot

31. Clip ductus

32. Cut ductus

33. Dissect arteria

34. Clip arteria

35. Cut arteria

(F) Retrograde cholecystectomy and terminate the procedure

36. Pull GB bed by means of tightened GB and last check GB bed

37. Dissect GB

38. Park GB on liver

39. Check hemostasis

40. Detach GB
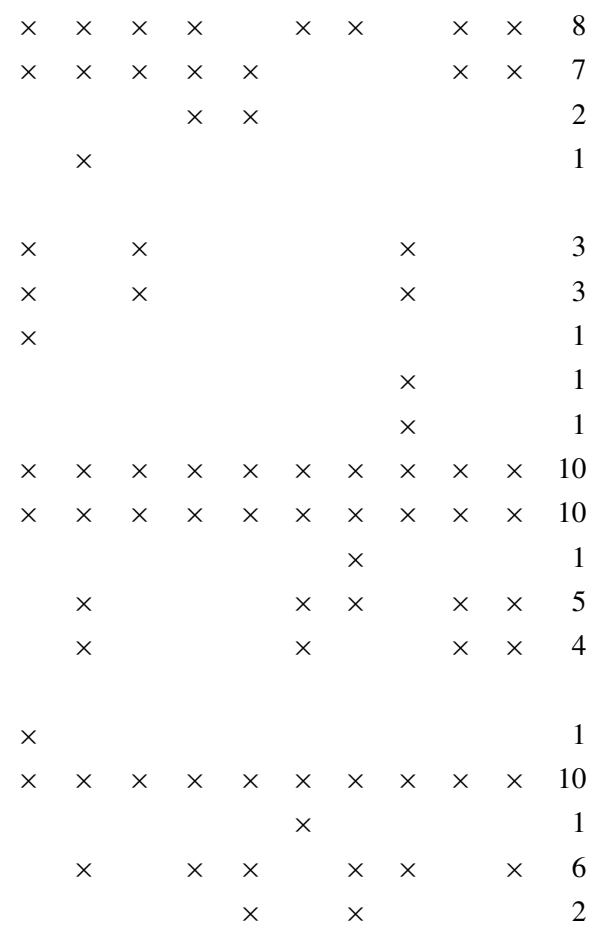
Table 2 continued

\begin{tabular}{lrllll}
\hline Steps & A1 A2 A3 A4 T1 T2 T3 T4 C1 C2 Identical steps \\
(no.)
\end{tabular}

GB: gallbladder; arteria: cystic artery; ductus: cystic duct; $\Delta$ Calot: Calot's triangle; CVS: critical view of safety

In step $D$ (mobilizing the infundibulum and "critical view of safety"), protocol A1 used the term "critical view of safety" of Strasberg [6, 10]. Seven protocols used the term Calot's triangle (A2-4, T1, T3-4, C1) but did not describe complete dissection of Calot's triangle; the other protocols did not mention these terms. The identification and dissection of the duct and artery was described by most protocols (duct: A1-4, T2-3, C1-2; artery: A1-4, T1, C12).

The main difference in step $E$ (clipping and cutting the cystic artery and duct) was the order for clipping these structures. Protocols A1, A3, and T4 clipped and cut the cystic artery first and then the cystic duct. A2, T2-3, and C1-2 described clipping and cutting the cystic duct first. Protocols A4 and T1 did not emphatically described clipping and cutting the cystic artery. The level of detail for clipping the duct also differed. Protocol A3 only described clipping the duct, protocol T2 included the number of clips, and five protocols also described the location of these clips (A1-2, T1, C1-2). Furthermore, the location description showed a difference in terminology used: " $2 \times$ central," " $2 \times$ distal, $1 \times$ toward gallbladder," " $2 \times$ choledochus side, $1 \times$ gallbladder side," " $2 \times$ central, $1 \times$ peripheral," and " $2 \times$ proximal, $1 \times$ distal." The level of detail also differed for clipping the artery. A1-3 only described clipping the artery; protocols T2 and T4 included the number of clips; and three protocols also described the location of these clips (T3, C1-2). Again, different terminology was used for the location description: " $2 \times$ arteria hepatica side, $1 \times$ gallbladder side," " $2 \times$ central, $1 \times$ peripheral," and " $2 \times$ proximal, $1 \times$ distal."

Finally, in step $F$ (retrograde cholecystectomy and terminating procedure) all protocols described dissecting the gallbladder. Here, different terminology was also used; A1, A3-4, T2, T4, and C2 described dissecting the gallbladder from the liver bed. $\mathrm{T} 1$ and $\mathrm{C} 1$ described subserous dissection of the gallbladder from the liver bed, and A2 noted antegrade removal and T3 retrograde removal of the gallbladder. Before disconnecting the gallbladder, seven protocols (A1-2, A4, T1, T3-4, C2) described checking the gallbladder bed and hemostasis. A3 and C1 described the check after disconnecting the gallbladder. All protocols described the removal of the gallbladder. A3, T2, and T4 described removal under vision; and protocols A1-2, T1-4, and $\mathrm{C} 1$ indicated removal via the umbilical trocar opening. Protocols A4 and T1-3 only described removal of the trocars under vision, whereas T2 and T3 also described the desufflation. Conversely, C2 described the desufflation first and removal of the trocars next. T2 removed three trocars first, desufflated via the last remaining 10-mm trocar, and removed this trocar afterward. None of the protocols described a checkup for bleeding at the trocar sites after their removal.

\section{Discussion}

Many differences exist in the studied Dutch surgical protocols for laparoscopic cholecystectomy. Currently, no standard for the surgical protocol is available in The Netherlands. Two hospitals, T5 and C3, did not even have a protocol, although it is compulsory for teaching hospital T5.

The protocols differ too much to be transferable, which if tried would lead to a lack of clarity. One of the differences concerns the definition of a protocol. Several documents are known and used as a protocol, which explains receiving both operative reports as well as instructions in this study. Although the influence of the lack of a uniform standardized protocol for laparoscopic cholecystectomy has not been determined scientifically, in the sense of a greater incidence of poor outcome, this lack of standardization in general and its influence has been proven in high-risk industries. Already in health care, several associations use protocols and guidelines for clinical decision making, for facilitating relevant training of the operating team, and as support for maintaining professional standards in daily practice $[13,21-23]$. One of the merits of 
standardizing the operative process in general and individual protocols in particular is improved communication among members of the operating team and between physicians and technicians by avoiding confusion with regard to the procedure's technical details (tasks and direction). Furthermore, "man-machine" interaction (communication between members of the operating team and the instruments and apparatus) can also be improved by using these protocols. Standardization also forms the basis for further use of the information and communication technology necessary for digitizing patient data, such as the use of the electronic medical record and the digital operative report. In addition, one standardized protocol, in combination with increasing surgical experience, can lead to a lower conversion rate during laparoscopic cholecystectomy [24].

This study gives a good representation of the current status of protocols used in academic hospitals (four of eight academic hospitals responded). These protocols mostly described the actions step by step. One advantage of a protocol is the detailed definition of actions, step by step, serving as a checklist comparable to those used in aviation. Also, without checking each action, the protocol diminishes the possibility of skipping important actions such as control of port-site bleeding after trocar removal [7, 13, 14].

The content of the protocols also differed. Although not emphasized in this study, it was noted that most protocols described the required instruments and equipment (A1, A3-4, T1-4, C2). By including equipment in the standardized protocol, a thorough preoperative setup can be established, thereby reducing the total operating time (no waiting for missing equipment) [19]. Warnings, instructions, and additional explanations during critical stages in the operation, such as instructions for adequate dissection of Calot's triangle, "critical view of safety," and instruction to prevent gallbladder perforations (resulting in bile leakage and stone spillage, possibly leading to complications) were not included in all protocols (A3, T2, C1-2) [1, $2,6,10]$. Including these items contributes to the completeness of the standardized protocol.

With regard to unambiguous language, it was concluded that different terminology was used and that the steps differed in their level of detail. This is mainly the case for clipping and cutting both the cystic duct and artery and the introduction of trocars, for which the location and introduction angles are of importance to prevent injury to organs and to create optimal working conditions [25-27]. However, scant literature is available that emphasizes the problems associated with poorly placed trocars [27]. Adequate trocar placement should provide direct access to target organs, optimal vision, decreased mental fatigue, and cognition of pathology and anatomy [27]. The position and size of the trocars varies among institutions and surgeons. For most standard techniques, the optical trocar is placed in the periumbilical region and the epigastric trocar in the epigastric region. These positions correspond to the results found. However, different locations for the working trocars can be found in the literature. In 2004, Ferzli and Fingerhut described placing these trocars in the right upper quadrant with one trocar parallel to the common bile duct, whereas Websurg (accessed April 2007) describes placing the working trocars left laterally to the umbilicus and in the right iliac fossa [27, 28]. Protocol C2 used the positions indicated by Websurg, and all other protocols used the standard fourtrocar technique indicated by Ferzli et al. [27].

The use of intraoperative cholangiography (IOC) to prevent common bile duct injury during laparoscopic cholecystectomy and routine versus selective IOC are still matters of debate [7, 9, 10, 29-32]. Injuries can occur despite the use of IOC, so it is not a precondition for safe performance $[10,11,33]$. Although our study did not focus on this aspect of laparoscopic cholecystectomy, it should be stated that none of the protocols described routine IOC. However, five protocols mentioned and described the use of selective IOC (A2-4, T3, C2). Both selective and routine IOC requires special expertise; and as yet surgeons in The Netherlands are not being trained for this technique.

Until now, the effect of introducing and using protocols regarding the safety of the procedure and the related bile duct injury is difficult to determine: first because of the absence of an implementation date (only five of ten protocols mentioned the edition date) and second because complication rates are not yet openly available in The Netherlands. With the introduction of a uniform protocol, the relation between the use of such a protocol and complication rates (e.g., bile duct injury) could be studied systematically.

International societies such as the European Association of Endoscopic Surgery (EAES) and the Society of American Gastrointestinal and Endoscopic Surgeons (SAGES) also provide guidelines for laparoscopic cholecystectomy $[9,34]$. Both societies' guidelines describe dissection in Calot's triangle using the "critical view" technique: The cystic duct and cystic artery must be identified clearly prior to clipping and cutting.

Although this study focused on the actions from trocar introduction to removal, preoperative and postoperative management (including establishing the pneumoperitoneum) should also be included in the future standardized protocol.

The authors propose that a uniform standardized protocol based on the "critical view of safety" principle (including complete dissection of Calot's triangle) be used that communicates through unambiguous steps and language. Standardization is no longer a matter to be left to personal preference. Implementation of a uniform 
Fig. 3 Summary of the steps of the Dutch protocol: "Best Practice: The Technique of Laparoscopic Cholecystectomy (CVS) by the Taskforce for Endoscopic Surgery of the Dutch Society of Surgery

(English translation)
Step 1 Preparation of the operation (not included)

\section{Step 2 Trocar positions}

Optical trocar

Access: caudally to the umbilicus. In case of obesity with a caudally situated umbilicus: optical trocar access cranially to the umbilicus.

Epigastric trocar

Two fingers caudally to the xyphoid process, at the right to the teres and falciform ligaments.

Two working trocars

At the level of the right upper quadrant of the abdomen.

Step 3 Abdominal exploration

Examine the condition of the gallbladder (adhesions, acute and/or chronic inflammation) and the liver (steatosis, cirrosis). Identification of Rouvière's sulcus is helpful.

\section{Step 4 Opening of peritoneal envelope}

Retraction of gallbladder

To open the peritoneal envelope the gallbladder must be adequately retracted. Pull the gallbladder's fundus cranially over the liver. After placing a clamp on the infundibulum (neck, Hartmann's pouch), retract caudo-ventrally.

Location for opening of peritoneal envelope

Both on the left and right side of the gallbladder the peritoneum directly on the neck of the gallbladder must be opened. Then the gallbladder's peritoneum is stripped off carefully.

Step 5 Mobilisation of the neck and Critical View of Safety (CVS)

Mobilise the neck of the gallbladder up to approximately $1 / 3$ of the gallbladder length from the gallbladder-bed of the liver. The supposed cystic duct and cystic artery are cleared circumferentially. Only after these two steps, in which two 'windows' are established ('two window technique') CVS is established. Not before this maneuver the cystic duct and the cystic artery are to be clipped.

Imaging and CVS

It is recommended to record CVS prior to transection of the cystic artery and cystic duct.

\section{Step 6 Cutting of cystic artery and duct}

Cystic artery

The cystic artery (diameter $<1 \mathrm{~mm}$ ) is preferably clipped and cut prior to clipping and cutting of the cystic duct. This measure also simplifies the view on Calot's triangle (increase of Calot's triangle up to $20 \%$ ) If the cystic duct is clipped and cut prior to clipping and cutting of the cystic artery, the artery can get torn by traction on the gallbladder.

Cystic duct

The cystic duct is clipped (two central clips) and cut after double check of CVS and Rouvière's sulcus. With regard to the central stump a large $\operatorname{rim}(\geq 1 \mathrm{~mm})$, peripherally to the clips, should be respected to limit the possibility of sliding off the clips.

Step 7 Retrograde cholecystectomy and termination of procedure

Prior to disconnecting the gallbladder a final check of haemostasis with regard to the gallbladder-bed must be made. Preferably remove the gallbladder, after replacing the laparoscope to the subxyphoidal port, through the optical trocar port site. The fascia of the two port sites with a diameter $\geq 10 \mathrm{~mm}$ must be closed to prevent hernias. standardized protocol can best be accomplished by the endorsement of an acknowledged association, such as a national scientific association or international society (e.g., the EAES and SAGES). Each time these associations distribute guidelines, a standardized protocol (including actions described step by step) should be added that can be used directly and without interpretation in the operating room. A future standardized protocol should also include patient data and indicate which data are to be recorded at what time [18]. Such an organized protocol can improve postoperative reporting and make it less time-consuming.

After having studied Dutch surgical protocols for laparoscopic cholecystectomy in this study and the protocols used in other fields of industry (e.g., aviation), the first version of a new protocol was drawn up by the Taskforce for Endoscopic Surgery of the Dutch Society of Surgery (Fig. 3) [35]. Since November 2006, this protocol is the certified and officially collated protocol for laparoscopic cholecystectomy in The Netherlands. It recommends image recording (analogue or digital) of the "critical view of safety" prior to cutting the duct and artery [12]. Image recordings are of interest for postoperative reporting and for understanding and treating possible complications. Furthermore, the recordings can contribute to the education of the operating team members. Finally, the Dutch Health Care Inspectorate recently announced that it would adopt this advice for their quality standard for laparoscopic cholecystectomy. 
Open Access This article is distributed under the terms of the Creative Commons Attribution Noncommercial License which permits any noncommercial use, distribution, and reproduction in any medium, provided the original author(s) and source are credited.

\section{References}

1. Gouma DJ, Rauws EA, Lameris JS (2004) Bile duct injury after cholecystectomy: risk of mortality substantially higher. [Galwegletsel na cholecystectomie: sterk verhoogde kans op sterfte.] Ned Tijdschr Geneeskd 148:1020-1024

2. Janssen IMC, Swank DJ, Boonstra O, et al (2003) Randomized clinical trial of ultrasonic versus electrocautery dissection of the gallbladder in laparoscopic cholecystectomy. Br J Surg 90: 799-803

3. Schol FPG, Go PMNYH, Gouma DJ (1994) Risk analysis for bile duct injury in laparoscopic cholecystectomy: analysis of 49 cases. Br J Surg 81:1786-1788

4. de Reuver PR, Rauws EA, Bruno MJ, et al (2007) Survival in bile duct injury patients after laparoscopic cholecystectomy: a multidisciplinary approach of gastroenterologists, radiologists, and surgeons. Surgery 142:1-9

5. Connor S, Garden OJ (2006) Bile duct injury in the era of laparoscopic cholecystectomy. Br J Surg 93:158-168

6. Strasberg SM (2002) Avoidance of biliary injury during laparoscopic cholecystectomy. J Hepatobiliary Pancreat Surg 9:543-547

7. Hugh TB (2002) New strategies to prevent laparoscopic bile duct injury-surgeons can learn from pilots. Surgery 132:826-835

8. Callery MP (2006) Avoiding biliary injury during laparoscopic cholecystectomy: technical considerations. Surg Endosc 20:1654-1658

9. Kuwada T (2005) Highlights of The Society of American Gastrointestinal and Endoscopic Surgeons 2005 Annual Meeting, April 2005, Ft. Lauderdale, FL. Medscape General Surgery, May (http://www.medscape.com/generalsurgery)

10. Strasberg SM, Hertl M, Soper NJ (1995) An analysis of the problem of biliary injury during laparoscopic cholecystectomy. J Am Coll Surg 180:101-125

11. Way LW, Stewart L, Gantert W, et al (2003) Causes and prevention of laparoscopic bile duct injuries: analysis of 252 cases from a human factors and cognitive psychology perspective. Ann Surg 237:460-469

12. Plaisier PW, Pauwels MMA, Lange JF (2001) Quality control in laparoscopic cholecystectomy: operation notes, video or photo print? HPB Surg 3:197-199

13. Kohn LT, Corrigan JM, Donaldson MS (2000) To err is human: building a safer health system. National Academy Press, Washington DC

14. Degani A, Wiener EL (1993) Cockpit checklists: concepts, design and use human factors. Hum Factors 35:345-359

15. Undre S, Sevdalis N, Healey AN, et al (2006) Teamwork in the operating theatre: cohesion or confusion? J Eval Clin Pract 12:182-189
16. Healey AN, Undre S, Vincent CA (2006) Defining the technical skills of teamwork in surgery. Qual Saf Health Care 15:231-234

17. Alarcon A, Berguer R (1996) A comparison of operating room crowding between open and laparoscopic operations. Surg Endosc 10:916-919

18. Cleary K, Kinsella A (2004) OR2020: the operating room of the future. Turf Valley Conference Center, Ellicott City, MD

19. Verdaasdonk EG, Stassen LP, van der Elst M, et al (2007) Problems with technical equipment during laparoscopic surgery: an observational study. Surg Endosc 21:275-279

20. Wauben LSGL, Van Veelen MA, Gossot D, et al (2006) Application of ergonomic guidelines during minimally invasive surgery: a questionnaire amongst 284 surgeons. Surg Endosc 20:1268-1274

21. Society of American Gastrointestinal and Endoscopic Surgeons (2007) http://www.sages.org/publications.html\#guide/. Accessed September 6, 2007

22. American College of Cardiology (2007) http://www.acc.org/. Accessed September 6, 2007

23. National Guideline Clearinghouse (2007) http://www. guideline.gov/. Accessed September 6, 2007

24. Gouma DJ (2006) Conversion from laparoscopic to open cholecystectomy. Br J Surg 93:905-906

25. Chandler JG, Voyles CR, Floore TL, et al (1997) Litigious consequences of open and laparoscopic biliary surgical mishaps. J Gastrointest Surg 1:138-145

26. Bhoyrul SB, Vierra MA, Nezhat CR, et al (2001) Trocar injuries in laparoscopic surgery. J Am Coll Surg 192:677-683

27. Ferzli GS, Fingerhut A (2004) Trocar placement for laparoscopic abdominal procedures: a simple standardized method. J Am Coll Surg 198:163-173

28. WebSurg (2007) http://www.websurg.com/. Accessed April 26, 2007

29. Nickkholgh A, Soltaniyekta S, Kalbasi H (2006) Routine versus selective intraoperative cholangiography during laparoscopic cholecystectomy: a survey of 2,130 patients undergoing laparoscopic cholecystectomy. Surg Endosc 20:868-874

30. Archer SB, Brown DW, Smith CD, et al (2001) Bile duct injury during laparoscopic cholecystectomy: results of a national survey. Ann Surg 234:549-559

31. Metcalfe MS, Ong T, Bruening MH, et al (2004) Is laparoscopic intraoperative cholangiogram a matter of routine? Am J Surg 187:475-481

32. Wu SC, Chen FC, Lo CJ (2005) Selective intraoperative cholangiography and single-stage management of common bile duct stone in laparoscopic cholecystectomy. World J Surg 29:1402-1408

33. Wright KD, Wellwood JM (1998) Bile duct injury during laparoscopic cholecystectomy without operative cholangiography. $\mathrm{Br}$ J Surg 85:191-194

34. Neugebauer EAM, Sauerland S, Fingerhut A, et al (2006) EAES guidelines for endoscopic surgery. Springer, Berlin

35. Dutch Society of Surgery. (http://nvvh.artsennet.nl/content/ resources/AMGATE_6059_635_TICH_L140905495/AMGATE_ 6059_635_TICH_R166206775948344//. Accessed May 3, 2007 